TRANSACTIONS OF THE

AMERICAN MATHEMATICAL SOCIETY

Volume 355, Number 1, Pages 57-78

S 0002-9947(02)03122-7

Article electronically published on August 21, 2002

\title{
INVERSE PROBLEM FOR UPPER ASYMPTOTIC DENSITY
}

\author{
RENLING JIN
}

\begin{abstract}
For a set $A$ of natural numbers, the structural properties are described when the upper asymptotic density of $2 A+\{0,1\}$ achieves the infimum of the upper asymptotic densities of all sets of the form $2 B+\{0,1\}$, where the upper asymptotic density of $B$ is greater than or equal to the upper asymptotic density of $A$. As a corollary, we prove that if the upper asymptotic density of $A$ is less than 1 and the upper asymptotic density of $2 A+\{0,1\}$ achieves the infimum, then the lower asymptotic density of $A$ must be 0 .
\end{abstract}

\section{INTRODUCTION}

Let $\mathbb{N}$ be the set of all natural numbers, including 0 . Let $A$ and $B$ always denote the subsets of $\mathbb{N}$, and let $a, b, c, i, j, k, l, m, n, x, y, z$ always denote the elements of $\mathbb{N}$. For any $m, n$ and any $A$, we write $[m, n]$ exclusively for the interval of integers $\{k \in \mathbb{N}: m \leqslant k \leqslant n\}$ and write $A(m, n)$ for the number of elements in $A \cap[m, n]$. For any $A$ and $B$, we write $A \pm B$ for the set $\{a \pm b: a \in A$ and $b \in B\}$. We write $2 A$ for $A+A$. For a set $A$ and a number $b$, we write $A \pm b$ for $A \pm\{b\}$. For a set $A$, the upper asymptotic density of $A$ is defined as

$$
\bar{d}(A)=\limsup _{n \rightarrow \infty} \frac{A(1, n)}{n} .
$$

The main results of the paper describe the structural properties of $A$ when

$$
\bar{d}(2 A+\{0,1\})=\inf \{\bar{d}(2 B+\{0,1\}): B \subseteq \mathbb{N} \text { and } \bar{d}(B) \geqslant \bar{d}(A)\} .
$$

Before the proof, we would like to have a brief introduction of the history of the subject.

For any set $A$, the Shnirel'man density $\sigma(A)$, the lower asymptotic density $\underline{d}(A)$, and the upper Banach density $B D(A)$ of $A$ are defined as follows:

$$
\begin{gathered}
\sigma(A)=\inf _{n \geqslant 1} \frac{A(1, n)}{n}, \\
\underline{d}(A)=\liminf _{n \rightarrow \infty} \frac{A(1, n)}{n}, \\
B D(A)=\lim _{n \rightarrow \infty} \sup _{m-k=n} \frac{A(k, m)}{m-k+1} .
\end{gathered}
$$

Received by the editors July 1, 2001 and, in revised form, May 8, 2002.

2000 Mathematics Subject Classification. Primary 11B05, 11B13, 11U10, 03 H15.

Key words and phrases. Upper asymptotic density, inverse problem, nonstandard analysis.

The author was supported in part by the NSF grant DMS-\#0070407. 
It is easy to see that for any set $A$, one has

$$
0 \leqslant \sigma(A) \leqslant \underline{d}(A) \leqslant \bar{d}(A) \leqslant B D(A) \leqslant 1 .
$$

Among density problems in additive number theory, people have been interested in finding the lower bound of the size of $A+B$ in terms of the sizes of $A$ and $B$. In 1930, Shnirel'man proved that if $0 \in A$, then $\sigma(A+B) \geqslant \sigma(A)+\sigma(B)-\sigma(A) \sigma(B)$ 2, page 3]. In 1942, Mann proved that if $0 \in A \cap B$, then $\sigma(A+B)=1$ or $\sigma(A+B) \geqslant \sigma(A)+\sigma(B)$ [2, page 5]. Mann's theorem was later generalized by Dyson [2, page 22] for considering the sum of more than two sets. Mann's theorem no longer holds if one replaces $\sigma$ by $\underline{d}$. For example, if both $A$ and $B$ are the set of all even numbers, then the lower asymptotic densities of $A, B$, and $A+B$ are all $\frac{1}{2}$. In 1950, Kneser proved a profound theorem similar to Mann's about lower asymptotic density [2 page 51]. For simplicity, I would like to state a special case of Kneser's theorem, which says that if $\underline{d}(A+B)<\underline{d}(A)+\underline{d}(B)$, then there exists a $k$ such that $A+B$ is a finite union of arithmetic sequences with a common difference $k$ and $\underline{d}(A+B) \geqslant \underline{d}(A)+\underline{d}(B)-\frac{1}{k}$.

Recently, the author discovered that the behavior of upper Banach density is very similar to that of Shnirel'man density and lower asymptotic density, while the behavior of upper asymptotic density is not. One theorem about upper Banach density parallel to Mann's theorem is proven in 5 and another theorem about upper Banach density parallel to Kneser's theorem is proven in [6]. The one parallel to Mann's theorem says that for any $A$ and $B$, one has that either $B D(A+B+\{0,1\})=$ 1 or $B D(A+B+\{0,1\}) \geqslant B D(A)+B D(B)$. The reason for adding the term $\{0,1\}$ is to avoid the trivial counterexample of both $A$ and $B$ being arithmetic sequences with the same common difference. For demonstrating the differences between the behavior of upper asymptotic density and the behavior of Shnirel'man density and lower asymptotic density, an example is given in [5]. In the example, two sets $A$ and $B$ are constructed so that for any $k$, one has $\bar{d}(A+B+[0, k])=\bar{d}(A)=\bar{d}(B)=\frac{1}{2}$. In that example, one can find that the upper asymptotic density of $A+B+[0, k]$ does not grow to be more than that of $A$ and $B$ because $A$ and $B$ achieve their upper asymptotic density at different paces. So the natural question to ask now is what is the lower bound of $\bar{d}(A+B+\{0,1\})$ when $A=B$.

During the DIMACS workshop "Unusual Applications of Number Theory" in January, 2000, Freiman showed the author a result about the upper asymptotic density of $2 A$. He proved the following theorem. Assume $0 \in A$ and $\operatorname{gcd}(A)=1$. Then $\bar{d}(2 A) \geqslant \frac{3}{2} \bar{d}(A)$ if $\bar{d}(A)<\frac{1}{2}$, and $\bar{d}(2 A) \geqslant \frac{1+\bar{d}(A)}{2}$ if $\bar{d}(A) \geqslant \frac{1}{2}$. This result is in fact an easy consequence of one of Freiman's theorems [8, Theorem 1.15, page 28]. Since Freiman's theorem will be needed in our proofs, we would like to state it as a lemma below.

Lemma 1.1 (G. Freiman). Let $A=\left\{a_{0}, a_{1}, \ldots, a_{k-1}\right\}$ be such that $0=a_{0}<a_{1}<$ $\cdots<a_{k-1}=n$ and $\operatorname{gcd}(A)=1$. If $k \leqslant \frac{n+3}{2}$, then $(2 A)(0,2 n) \geqslant 3 k-3$. If $k \geqslant \frac{n+3}{2}$, then $(2 A)(0,2 n) \geqslant k+n$.

For convenience, we would like to point out a weaker form of Freiman's result above about upper asymptotic density.

For any set $A$ with $\bar{d}(A)=\alpha$, one has that $\bar{d}(2 A+\{0,1\}) \geqslant \frac{1+\alpha}{2}$ if $\alpha \geqslant \frac{1}{2}$, and $\bar{d}(2 A+\{0,1\}) \geqslant \frac{3}{2} \alpha$ if $\alpha<\frac{1}{2}$.

Note that the inequalities cannot be improved by the following example. For each real number $r$, let $\lceil r\rceil$ denote the smallest integer greater than or equal to $r$. 
Example 1.2. For any real number $\alpha$ with $0 \leqslant \alpha \leqslant 1$, let

$$
A=\bigcup_{n=1}^{\infty}\left[\left\lceil(1-\alpha) 2^{2^{n}}\right\rceil, 2^{2^{n}}\right]
$$

Then $\bar{d}(A)=\alpha, \bar{d}(2 A+\{0,1\})=\frac{1+\alpha}{2}$ if $\alpha \geqslant \frac{1}{2}$, and $\bar{d}(2 A+\{0,1\})=\frac{3}{2} \alpha$ if $\alpha<\frac{1}{2}$.

It is usually the case that after the lower bound of the size of $A+B$ is found, one wants to consider the inverse problem of what conclusion can be drawn when the lower bound is achieved. For example, the Shnirel'man pairs and Mann pairs are considered in [3]. A general idea developed by Freiman and others says that if $2 A$ is "small", then $A$ must possess some structure. In fact, Kneser's theorem is a witness of this idea with $2 A$ being replaced by $A+B$. A variation of Kneser's theorem for $2 A$ proved in [1] is another witness of the idea. There are also many other witnesses of the idea for finite sets. For example, if $A$ is finite and if $|2 A|=2|A|-1$, then $A$ is an arithmetic progression [8, Theorem 1.2, page 6]. Freiman proved that if $A$ is finite and $|2 A| \leqslant 3|A|-4$, then $A$ is a subset of an arithmetic progression of length less than or equal to $2|A|-3$. Following the same idea, we want to consider the inverse problem for upper asymptotic density. We describe the structural properties of $A$ when the upper asymptotic density of $2 A+\{0,1\}$ achieves its infimum. The following is the main theorem of the paper.

Theorem 1.3. Let $A$ be a set of natural numbers.

Part I: Assume $\bar{d}(A) \geqslant \frac{1}{2}$. Then $\bar{d}(2 A+\{0,1\})=\frac{1+\bar{d}(A)}{2}$ implies that for any increasing sequence $\left\langle b_{n}: n \in \mathbb{N}\right\rangle$ with $\lim _{n \rightarrow \infty} \frac{A\left(0, b_{n}\right)}{b_{n}+1}=\bar{d}(A)$, one has

$$
\lim _{n \rightarrow \infty} \frac{(2 A+\{0,1\})\left(0, b_{n}\right)}{b_{n}+1}=\bar{d}(A) .
$$

Part II: Assume $0<\bar{d}(A)<\frac{1}{2}$. Then $\bar{d}(2 A+\{0,1\})=\frac{3}{2} \bar{d}(A)$ implies that for any increasing sequence $\left\langle b_{n}: n \in \mathbb{N}\right\rangle$ with $\lim _{n \rightarrow \infty} \frac{A\left(0, b_{n}\right)}{b_{n}+1}=\bar{d}(A)$, there exist two sequences $\left\langle a_{n}: n \in \mathbb{N}\right\rangle$ and $\left\langle c_{n}: n \in \mathbb{N}\right\rangle$ such that

$$
\begin{gathered}
\lim _{n \rightarrow \infty} \frac{A\left(a_{n}, b_{n}\right)}{b_{n}-a_{n}+1}=1, \\
\lim _{n \rightarrow \infty} \frac{c_{n}}{b_{n}}=0,
\end{gathered}
$$

and $\left[c_{n}, a_{n}-1\right] \cap A=\emptyset$ for every $n \in \mathbb{N}$.

One can derive an interesting corollary from Theorem 1.3 ,

Corollary 1.4. Assume $\bar{d}(A)<1$. If

$$
\bar{d}(2 A+\{0,1\})=\inf \{\bar{d}(2 B+\{0,1\}): B \subseteq \mathbb{N} \text { and } \bar{d}(B) \geqslant \bar{d}(A)\},
$$

then $\underline{d}(A)=0$.

\section{LEMMAS}

We need more lemmas in the proof of Theorem 1.3. Nonstandard methods will be used in and after Lemma 2.4 The next lemma is a consequence of Besicovitch's theorem. Besicovitch's theorem says the following [2, page 6]. Let $\alpha$ and $\beta$ be two nonnegative real numbers such that $\alpha+\beta \leqslant 1$. Suppose $1 \in A, 0 \in B, \sigma(A)=\alpha$, and $B(1, m) \geqslant \beta(m+1)$ for every $m>0$. Then $\sigma(A+B) \geqslant \alpha+\beta$. Note that in 
Besicovitch's theorem, whether or not a number $m>n$ is in $B$ and whether or not a number $m>n+1$ is in $A$ are irrelevant to the value of $\frac{(A+B)(1, n+1)}{n+1}$.

Lemma 2.1. Let $\alpha$ and $\beta$ be two nonnegative real numbers such that $\alpha+\beta \leqslant 1$. Let $A \subseteq[a, a+n]$ and $B \subseteq[b, b+n]$ be such that $a \in A, b \in B$,

$$
\frac{A(a, a+m)}{m+1} \geqslant \alpha, \text { and } \frac{B(a, a+m)}{m+1} \geqslant \beta
$$

for $m=0,1, \ldots, n$. Then

$$
\frac{(A+B+\{0,1\})(a+b, a+b+m)}{m+1} \geqslant \alpha+\beta
$$

for $m=0,1, \ldots, n$.

Proof. Without loss of generality, we can assume that $\beta \leqslant \frac{1}{2}$. We can also assume that $a=1$ and $b=0$ because otherwise we can replace $A$ by $A-a+1$, replace $B$ by $B-b$ and apply the following equality:

$$
\begin{aligned}
& (A+B+\{0,1\})(a+b, a+b+m) \\
& \quad=((A-a+1)+(B-b)+\{0,1\})(1,1+m) .
\end{aligned}
$$

Let $B^{\prime}=B+\{0,1\}$. The lemma is trivially true if $B=[0, n]$. So we assume that $[0, n] \backslash B \neq \emptyset$ and let $k_{0}=\min ([0, n] \backslash B)$.

Case $1: B=\left[0, k_{0}-1\right]\left(\right.$ so $\left.B^{\prime}=\left[0, k_{0}\right]\right)$. We prove the lemma by induction on $m$.

For $m=0,1, \ldots, k_{0}-1$, one has

$$
\frac{\left(A+B^{\prime}\right)(1,1+m)}{m+1} \geqslant \frac{B^{\prime}(1,1+m)}{m+1}=1 \geqslant \alpha+\beta .
$$

Suppose that $\frac{\left(A+B^{\prime}\right)(1,1+k)}{k+1} \geqslant \alpha+\beta$ for some $k \in\left[k_{0}, n-1\right]$.

Let $m=k+1$. If there is an $x \in A \cap\left[m+1-k_{0}, m+1\right]$, then there is a $y \in B^{\prime}=\left[0, k_{0}\right]$ such that $1+m=x+y \in A+B^{\prime}$. Hence one has

$$
\begin{aligned}
\frac{\left(A+B^{\prime}\right)(1,1+m)}{m+1}=\frac{\left(A+B^{\prime}\right)(1,1+k)+1}{m+1} \\
\quad=\frac{\left(A+B^{\prime}\right)(1,1+k)}{k+1} \cdot \frac{k+1}{m+1}+\frac{1}{m+1} \\
\geqslant(\alpha+\beta) \frac{k+1}{m+1}+(\alpha+\beta) \frac{1}{m+1} \\
=\alpha+\beta .
\end{aligned}
$$

Otherwise, let $x=\max (A \cap[1, m+1])$. So $x<m+1-k_{0}$ and one has that

$$
\begin{aligned}
\frac{\left(A+B^{\prime}\right)(1,1+m)}{m+1} & \\
& \geqslant \frac{A(1, x)+\left(x+B^{\prime}\right)\left(x+1, x+k_{0}\right)}{m+1} \\
& =\frac{A(1, m+1)}{m+1}+\frac{B^{\prime}(1, m+1)}{m+1} \\
& \geqslant \frac{A(1, m+1)}{m+1}+\frac{B(0, m)}{m+1} \\
& \geqslant \alpha+\beta .
\end{aligned}
$$

This ends the induction. 
Case 2: $B \neq\left[0, k_{0}-1\right] . \quad$ Let $k_{1}=\min \left(B \backslash\left[0, k_{0}-1\right]\right)$ (so $\left.k_{1} \in B^{\prime} \backslash(B+1)\right)$. By Besicovitch's theorem, one need only to show that $\frac{B^{\prime}(1, m)}{m+1} \geqslant \beta$ for $m=1,2, \ldots, n$. For $m \in\left[1, k_{0}\right]$, one has

$$
\frac{B^{\prime}(1, m)}{m+1}=\frac{m}{m+1} \geqslant \frac{1}{2} \geqslant \beta,
$$

for $m \in\left[k_{0}+1, k_{1}-1\right]$, one has

$$
\frac{B^{\prime}(1, m)}{m+1}=\frac{k_{0}}{m+1}=\frac{B(0, m)}{m+1} \geqslant \beta,
$$

and for $m \in\left[k_{1}, n+1\right]$, one has

$$
\frac{B^{\prime}(1, m)}{m+1} \geqslant \frac{(B+1)(1, m)+1}{m+1}=\frac{B(0, m-1)+1}{m+1} \geqslant \frac{B(0, m)}{m+1} \geqslant \beta .
$$

\section{$\square($ Lemma 2.1)}

The next lemma is another variation of Besicovitch's theorem.

Lemma 2.2. Let $\beta \geqslant 0$ be a real number and let $A \subseteq[a, a+n]$ and $B \subseteq[b, b+n]$ be such that $a \in A$ and $b \in B$. Suppose that for each $x \in[0, n], \frac{B(b, b+x)}{x+1} \geqslant \beta$. Suppose also that there is a sequence $a-1=x_{0}<x_{1}<\cdots<x_{k}=a+n$ and a sequence of nonnegative real numbers $r_{0}, r_{1}, \ldots, r_{k-1}$ such that for each $i \in[0, k-1], r_{i}+\beta \leqslant 1$ and for each $x \in\left[x_{i}+1, x_{i+1}\right]$,

$$
\frac{A\left(x_{i}+1, x\right)}{x-x_{i}} \geqslant \frac{A\left(x_{i}+1, x_{i+1}\right)}{x_{i+1}-x_{i}}=r_{i} .
$$

Then one has

$$
(A+B+\{0,1\})(a+b, a+b+n) \geqslant A(a, a+n)+(n+1) \beta .
$$

Proof. Without loss of generality, we assume that $a=b=0$. By Lemma 2.1, one has that for each $i \in[0, k-1]$,

$$
\begin{aligned}
\frac{(A+B+\{0,1\})\left(x_{i}+1, x_{i+1}\right)}{x_{i+1}-x_{i}} & \\
\geqslant & \frac{\left(A \cap\left[x_{i}+1, x_{i+1}\right]+B \cap\left[0, x_{i+1}-x_{i}-1\right]+\{0,1\}\right)\left(x_{i}+1, x_{i+1}\right)}{x_{i+1}-x_{i}} \\
& \geqslant r_{i}+\beta .
\end{aligned}
$$

Hence,

$$
\begin{aligned}
(A+ & B+\{0,1\})(0, n) \\
& =\sum_{i=0}^{k-1}(A+B+\{0,1\})\left(x_{i}+1, x_{i+1}\right) \\
& \geqslant \sum_{i=0}^{k-1}\left(r_{i}+\beta\right)\left(x_{i+1}-x_{i}\right) \\
& =\sum_{i=0}^{k-1} r_{i}\left(x_{i+1}-x_{i}\right)+\beta \sum_{i=0}^{k-1}\left(x_{i+1}-x_{i}\right) \\
& =A(0, n)+(n+1) \beta .
\end{aligned}
$$

This ends the proof of the lemma. $\square$ (Lemma 2.2) 
Lemma 2.3. Let $A \subseteq[a, b]$. Let $x_{0}=a-1$ and define a sequence $x_{0}<x_{1}<\cdots<$ $x_{k}=b$ inductively such that if $x_{i}<b$ is defined, then let

$$
x_{i+1}=\min \left\{x \in\left[x_{i}+1, b\right]: \frac{A\left(x_{i}+1, x\right)}{x-x_{i}}=\min \left\{\frac{A\left(x_{i}+1, y\right)}{y-x_{i}}: y \in\left[x_{i}+1, b\right]\right\}\right\} \text {. }
$$

For each $i \in[0, k-1]$ let $r_{i}=\frac{A\left(x_{i}+1, x_{i+1}\right)}{x_{i+1}-x_{i}}$. Then for each $i \in[0, k-2]$,

$$
r_{i} \leqslant r_{i+1}
$$

and for each $i \in[0, k-1]$ and $x \in\left[x_{i}+1, x_{i+1}\right]$,

$$
\frac{A\left(x_{i}+1, x\right)}{x-x_{i}} \geqslant r_{i} \text {. }
$$

Proof. The inequality $r_{i} \leqslant r_{i+1}$ is true because if $r_{i}>r_{i+1}$, then

$$
\begin{aligned}
\frac{A\left(x_{i}+1, x_{i+2}\right)}{x_{i+2}-x_{i}} & =\frac{A\left(x_{i}+1, x_{i+1}\right)+A\left(x_{i+1}+1, x_{i+2}\right)}{x_{i+2}-x_{i}} \\
& =\frac{r_{i}\left(x_{i+1}-x_{i}\right)+r_{i+1}\left(x_{i+2}-x_{i+1}\right)}{x_{i+2}-x_{i}}<r_{i},
\end{aligned}
$$

which contradicts the minimality of $r_{i}$. Also, for each $x \in\left[x_{i}+1, x_{i+1}\right], \frac{A\left(x_{i}+1, x\right)}{x-x_{i}} \geqslant$ $r_{i}$ by the minimality of $r_{i} . \quad \square$ (Lemma 2.3)

We would like to use methods from nonstandard analysis from now on. One of the advantages of nonstandard methods is that an asymptotic argument such as upper asymptotic density in the standard world can be translated into a *finite argument in a nonstandard universe. For basic knowledge of nonstandard analysis, the reader is recommended to consult [7], 4], or [5].

We fix an $\aleph_{1}$-saturated nonstandard universe ${ }^{*} V$ in this paper. For each set $A$, we write ${ }^{*} A$ for the nonstandard version of $A$ in ${ }^{*} V$. For example, ${ }^{*} \mathbb{N}$ is the set of all natural numbers in ${ }^{*} V$. For example also, if $A$ is the set of all even numbers in $\mathbb{N}$, then ${ }^{*} A$ is the set of all even numbers in ${ }^{*} \mathbb{N}$. We use $a, b, c, x, y, z$ for natural numbers in ${ }^{*} \mathbb{N}$ and reserve $l, m$ and $n$ for natural numbers in $\mathbb{N}$. The integers in $* \mathbb{N} \backslash \mathbb{N}$ are called hyperfinite integers, denoted sometimes by $H, K$ and $N$. We write $r, s$, and $t$ for both standard or nonstandard real numbers and reserve $\alpha, \beta, \gamma$, and $\epsilon$ for standard real numbers. We write $\iota$ for an infinitesimal, i.e., $-\frac{1}{n}<\iota<\frac{1}{n}$ for every standard positive integer $n$. We write $X, Y$ for internal subsets of *NN . Next we would like to prove a lemma showing how upper asymptotic density can be translated into a nonstandard version.

Lemma 2.4. Let $\alpha \geqslant 0$ and let $A$ be a set of standard natural numbers. Then $\bar{d}(A) \geqslant \alpha$ if and only if there is a hyperfinite integer $H$ and an infinitesimal $\iota$ such that

$$
\frac{{ }^{*} A(0, H)}{H+1} \geqslant \alpha-\iota .
$$

Proof. " $\Rightarrow "$ : Let $\left\langle b_{n}: n \in \mathbb{N}\right\rangle$ be an increasing sequence in $\mathbb{N}$ such that

$$
\lim _{n \rightarrow \infty} \frac{A\left(0, b_{n}\right)}{b_{n}+1}=\bar{d}(A) \geqslant \alpha .
$$

By passing down to a subsequence, one can assume, without loss of generality, that

$$
\frac{A\left(0, b_{n}\right)}{b_{n}+1} \geqslant \alpha-\frac{1}{n}
$$


for each $n \in \mathbb{N}$. By the overspill principle, one can find a hyperfinite integer $N$ such that

$$
\frac{{ }^{*} A\left(0, b_{N}\right)}{b_{N}+1} \geqslant \alpha-\frac{1}{N} .
$$

Now let $H=b_{N}$ and let $\iota=\frac{1}{N}$. Clearly, $H$ is hyperfinite and $\iota$ is an infinitesimal. " $\Leftarrow$ ": For each $n \in \mathbb{N}$, let $\varphi\left({ }^{*} \mathbb{N},{ }^{*} A, n, \alpha\right)$ be the following sentence:

$$
\left(\exists x \in{ }^{*} \mathbb{N}\right)\left(x>n \text { and } \frac{{ }^{*} A(0, x)}{x+1} \geqslant \alpha-\frac{1}{n}\right) .
$$

It is clear that $\varphi\left({ }^{*} \mathbb{N},{ }^{*} A, n, \alpha\right)$ is true in ${ }^{*} V$ because $H$ is a witness for the existential quantifier. By the transfer principle, we know that $\varphi(\mathbb{N}, A, n, \alpha)$ is true in the standard world for every $n \in \mathbb{N}$. That means $\bar{d}(A) \geqslant \alpha . \quad \square($ Lemma 2.4)

For the convenience of handling nonstandard arguments, we would like to introduce some notation of comparisons. For any real numbers $r, s$ in ${ }^{*} V$, by $r \approx s$ we mean that $r-s$ is an infinitesimal, by $r \ll s$ we mean that $r<s$ and $r \not \approx s$, and by $r \lesssim s(r \gtrsim s)$ we mean $r<s(r>s)$ or $r \approx s$. Given a hyperfinite integer $H$ and two natural numbers $a, b$, by $a \sim_{H} b$ we mean that $\frac{b-a}{H} \approx 0$, by $a \prec_{H} b$ we mean that $a<b$ and $a \chi_{H} b$, and by $a \preceq_{H} b\left(a \succeq_{H} b\right)$ we mean that $a \prec_{H} b\left(a \succ_{H} b\right)$ or $a \sim_{H} b$. We often say that $a$ is insignificant with respect to $H$ if $a \sim_{H} 0$. The subscript $H$ will be dropped when it is clear. Now we introduce more lemmas needed in the proof of the theorem. The next lemma is a nonstandard version of Lemma 2.1.

Lemma 2.5. Let $\alpha, \beta$ be two standard nonnegative real numbers such that $\alpha+\beta<$ 1. Let $H$ be a hyperfinite integer. Suppose $X \subseteq[a, a+H]$ and $Y \subseteq[b, b+H]$ such that $a \in X, b \in Y$, and for each $x$ with $0 \prec x \leqslant H$, one has

$$
\frac{X(a, a+x)}{x+1} \gg \alpha \text { and } \frac{Y(b, b+x)}{x+1} \gtrsim \beta .
$$

Then for each $x$ with $0 \prec x \leqslant H$,

$$
\frac{(X+Y+\{0,1\})(a+b, a+b+x)}{x+1} \gg \alpha+\beta .
$$

Proof. Without loss of generality, we assume $a=b=0$. We will use $a$ and $b$ for other purposes in the proof. Given a $c$ with $0 \prec c \leqslant H$, it suffices to show that

$$
\frac{(X+Y+\{0,1\})(0, c)}{c+1} \gg \alpha+\beta .
$$

Claim 2.5.1. There is a $z \sim 0$ such that for each $x \in[z, H]$, one has

$$
\frac{Y(z, x)}{x-z+1} \gtrsim \beta .
$$

Proof of Claim 2.5.1. Let $-1=x_{0}<x_{1}<\cdots<x_{k}$ and $r_{0}, r_{1}, \ldots, r_{k-1}$ be two sequences constructed as in Lemma 2.3 with $[a, b]$ being replaced by $[0, H]$ and $A$ being replaced by $Y$. For each $m \in \mathbb{N}$, let $i_{m} \in[0, k-1]$ be 0 if $r_{0}>\beta-\frac{1}{m}$ or the largest $i$ with $r_{i} \leqslant \beta-\frac{1}{m}$. Note that if $i_{m} \neq 0$, then $x_{i_{m}+1} \sim 0$ because otherwise 
one has

$$
\begin{aligned}
\frac{Y\left(0, x_{i_{m}+1}\right)}{x_{i_{m}+1}+1} & =\frac{\sum_{i=0}^{i_{m}} Y\left(x_{i}+1, x_{i+1}\right)}{x_{i_{m}+1}+1} \\
& =\frac{\sum_{i=0}^{i_{m}} r_{i}\left(x_{i+1}-x_{i}\right)}{x_{i_{m}+1}+1} \leqslant r_{i_{m}} \leqslant \beta-\frac{1}{m},
\end{aligned}
$$

which contradicts that $\frac{Y(0, x)}{x+1} \gtrsim \beta$ for each $x$ with $0 \prec x \leqslant H$. Since for each $m \in \mathbb{N}, x_{i_{m}+1} \leqslant x_{i_{m+1}+1} \sim 0$ and the set of all $x \sim 0$ has uncountable cofinality by $\aleph_{1}$-saturation, there is a hyperfinite integer $K$ such that for each hyperfinite $N<K, x_{i_{N}+1} \sim 0$. By $\aleph_{1}$-saturation again, one can find a hyperfinite $N<K$ and let $z=x_{i_{N}+1}+1$ such that for each $x \in[z, H]$,

$$
\frac{Y(z, x)}{x-z+1} \geqslant r_{i_{N}+1} \geqslant \beta-\frac{1}{N} \text {. }
$$

Hence,

$$
\frac{Y(z, x)}{x-z+1} \gtrsim \beta
$$

for each $x \in[z, H] . \quad \square($ Claim $2.5,1)$

Since $\frac{z}{H+1} \approx 0$, we can assume, without loss of generality, that $z=0$. (When $x \sim_{H} x^{\prime}$ and $y \sim_{H} y^{\prime}$, we often write $[x, y]$ for $\left[x^{\prime}, y^{\prime}\right]$ or vice versa for simplifying proofs because the difference between $\frac{Y(x, y)}{H+1}$ and $\frac{Y\left(x^{\prime}, y^{\prime}\right)}{H+1}$ is an infinitesimal.) Let $\iota$ be an infinitesimal such that for every $x \in[0, H], \frac{Y(0, x)}{x+1} \geqslant \beta-\iota$.

Let $\frac{X(0, c)}{c+1}=s \gg \alpha$ and let $t=\min \{s-\alpha, 1-\alpha-\beta\}$. Note that $t \gg 0$.

Claim 2.5.2. There is a $b \prec c$ such that for each $x \in[b, c], \frac{X(b, x)}{x-b+1} \gg \alpha$.

Proof of Claim 2.5.2. Let $-1=x_{0}<x_{1}<\cdots<x_{k}=c$ and $r_{0}, r_{1}, \ldots, r_{k-1}$ be two sequences constructed as in Lemma 2.3 with $[a, b]$ being replaced by $[0, c]$ and $A$ being replaced by $X \cap[0, c]$. Let $j$ be 0 if $r_{0} \geqslant \alpha+\frac{t}{2}$ or be the largest number in $[0, k-1]$ such that $r_{j} \leqslant \alpha+\frac{t}{2}$. Now $x_{j+1} \prec c$ because otherwise

$$
\frac{X(0, c)}{c+1} \approx \frac{X\left(0, x_{j+1}\right)}{x_{j+1}+1} \leqslant r_{j} \leqslant \alpha+\frac{t}{2},
$$

which contradicts $\frac{X(0, c)}{c+1}=s=\alpha+t \gg \alpha+\frac{t}{2}$. Let $b=x_{j+1}+1$. It is clear that for each $x \in[b, c]$,

by the choice of $j . \quad \square($ Claim 2.5.2)

$$
\frac{X(b, x)}{x-b+1} \geqslant \alpha+\frac{t}{2} \gg \alpha
$$

If $b \sim 0$, then we can assume, without loss of generality, that $b=0$. Let

$$
r=\min \left\{\frac{X(0, x)}{x+1}: x \in[0, c]\right\} .
$$

Then $r \gg \alpha$. Now the lemma follows from Lemma 2.1] because

$$
\frac{(X+Y+\{0,1\})(0, c)}{c+1} \geqslant r+\beta-\iota \gg \alpha+\beta .
$$

Hence we can assume that $0 \prec b \prec c$ in the rest of the proof of Lemma 2.5. 
Claim 2.5.3. $\frac{(X+Y+\{0,1\})(0, b)}{b+1} \gtrsim \alpha+\beta$.

Proof of Claim 2.5.3. Let $-1=x_{0}<x_{1}<\cdots<x_{k}=b$ and $r_{0}, r_{1}, \ldots, r_{k-1}$ be two sequences constructed as in Lemma 2.3 with $[a, b]$ being replaced by $[0, b]$ and $A$ being replaced by $X \cap[0, b]$. Given a standard positive real $\epsilon$, it suffices to show that

$$
\frac{(X+Y+\{0,1\})(0, b)}{b+1} \geqslant \alpha+\beta-\epsilon .
$$

Choose a $z$ with $0 \prec z \prec b$ such that

$$
\frac{z}{b+1} \leqslant \frac{\epsilon}{2(\alpha+\beta+1)}
$$

Let $j \in[0, k-1]$ be such that $x_{j}<z \leqslant x_{j+1}$. Then $r_{j} \gtrsim \alpha$ because otherwise

$$
\frac{X\left(0, x_{j+1}\right)}{x_{j+1}+1} \leqslant r_{j} \ll \alpha,
$$

which contradicts a condition of this lemma. Hence we have

$$
\begin{aligned}
\frac{(X+Y+\{0,1\})(0, b)}{b+1} \\
\geqslant \frac{(X+Y+\{0,1\})\left(x_{j}+1, b\right)}{b+1} \\
\geqslant \frac{\left(X \cap\left[x_{j}+1, b\right]+Y \cap\left[0, b-x_{j}-1\right]+\{0,1\}\right)\left(x_{j}+1, b\right)}{b+1} \\
\geqslant \frac{r_{j}\left(b-x_{j}\right)+(\beta-\iota)\left(b-x_{j}\right)}{b+1} \\
\geqslant\left(r_{j}+\beta-\iota\right)\left(1-\frac{x_{j}+1}{b+1}\right) \\
\geqslant\left(r_{j}+\beta-\iota\right)\left(1-\frac{\epsilon}{2(\alpha+\beta+1)}\right) \\
\geqslant r_{j}+\beta-\iota-\left(\frac{\epsilon}{2}\right)\left(\frac{r_{j}+\beta-\iota}{\alpha+\beta+1}\right) \\
\gtrsim r_{j}+\beta-\iota-\frac{\epsilon}{2} \\
>\alpha+\beta-\epsilon .
\end{aligned}
$$

\section{$\square($ Claim 2.5.3)}

Now we finish the proof of the lemma by showing that $\frac{(X+Y+\{0,1\})(0, c)}{c+1} \gg \alpha+\beta$. Following Claim 2.5.3, we have $0 \prec b \prec c$ and that there is an $s \gtrsim \alpha+\beta$ such that

$$
\frac{(X+Y+\{0,1\})(0, b)}{b+1} \geqslant s
$$

and there is a $t \gg \alpha$ such that

$$
\frac{X(b+1, x)}{x-b} \geqslant t
$$


for every $x \in[b+1, c]$. Hence by Lemma 2.1

$$
\begin{aligned}
& \frac{(X+Y+\{0,1\})(0, c)}{c+1} \\
& \quad=\frac{(X+Y+\{0,1\})(0, b)}{c+1}+\frac{(X+Y+\{0,1\})(b+1, c)}{c+1} \\
& \geqslant s \cdot \frac{b+1}{c+1}+\frac{(X \cap[b+1, c]+Y \cap[0, c-b-1]+\{0,1\})(b+1, c)}{c+1} \\
& \geqslant s \cdot \frac{b+1}{c+1}+(t+\beta-\iota) \cdot \frac{c-b}{c+1} \\
& \gg \alpha+\beta .
\end{aligned}
$$

$\square$ (Lemma 2.5)

Corollary 2.6. Let $\alpha, \beta$ be two standard nonnegative real numbers such that $\alpha+$ $\beta \leqslant 1$. Let $X \subseteq[a, a+H]$ and $Y \subseteq[b, b+H]$ be such that $a \in X, b \in Y$, and for each $x$ with $0 \prec x \leqslant H$,

$$
\frac{X(a, a+x)}{x+1} \gtrsim \alpha \text { and } \frac{Y(b, b+x)}{x+1} \gtrsim \beta .
$$

Then for each $x$ with $0 \prec x \leqslant H$,

$$
\frac{(X+Y+\{0,1\})(a+b, a+b+x)}{x+1} \gtrsim \alpha+\beta .
$$

Proof. Replace $\alpha$ by $\alpha-\frac{1}{n}$ in Lemma 2.5 and let $n \rightarrow \infty$. $\square$ (Corollary 2.6)

Next we introduce two lemmas, which translate two conclusions of Theorem 1.3 into nonstandard forms.

Lemma 2.7. Let $A$ be such that $\bar{d}(A)=\alpha \geqslant \frac{1}{2}$. Suppose that for each hyperfinite integer $H$ with $\frac{{ }^{*} A(0, H)}{H+1} \approx \alpha$, one has

$$
\frac{\left(2^{*} A+\{0,1\}\right)(0, H)}{H+1} \approx \alpha .
$$

Then the conclusion of Part I of Theorem 1.3 is true.

Proof. Let $\left\langle b_{n}: n \in \mathbb{N}\right\rangle$ be an increasing sequence in $\mathbb{N}$ such that

$$
\lim _{n \rightarrow \infty} \frac{A\left(0, b_{n}\right)}{b_{n}+1}=\alpha
$$

Then, by the transfer property, $\frac{{ }^{*} A\left(0, b_{N}\right)}{b_{N}+1} \approx \alpha$ for each hyperfinite $N$. Hence, by the assumption of the lemma, one has

$$
\frac{\left(2^{*} A+\{0,1\}\right)\left(0, b_{N}\right)}{b_{N}+1} \approx \alpha .
$$

This means that for each $k \in \mathbb{N}$ and each hyperfinite $K$, the sentence $\varphi\left({ }^{*} A,{ }^{*} \mathbb{N}, K, k,\left\langle b_{n}: n \in{ }^{*} \mathbb{N}\right\rangle\right)$ is true in ${ }^{*} V$, where

$$
\begin{aligned}
& \varphi\left({ }^{*} A,{ }^{*} \mathbb{N}, K, k,\left\langle b_{n}: n \in \mathbb{N}\right\rangle\right)= \\
& \quad\left(\forall N \in \mathbb{N}^{*} \mathbb{N}\right)\left(N>K \text { implies } \alpha+\frac{1}{k}>\frac{(2 * A+\{0,1\})\left(0, b_{N}\right)}{b_{N}+1}>\alpha-\frac{1}{k}\right) .
\end{aligned}
$$


By the underspill principle and the transfer principle, there exists an $l_{k} \in \mathbb{N}$ such that $\varphi\left(A, \mathbb{N}, l_{k}, k,\left\langle b_{n}: n \in \mathbb{N}\right\rangle\right)$ is true in the standard world. This means that as $k$ approaches $\infty$, one has

$$
\lim _{n \rightarrow \infty} \frac{(2 A+\{0,1\})\left(0, b_{n}\right)}{b_{n}+1}=\alpha
$$

$\square$ (Lemma 2.7)

Lemma 2.8. Let $A$ be such that $\bar{d}(A)=\alpha<\frac{1}{2}$. Suppose that for each hyperfinite integer $H$ with $\frac{{ }^{*} A(0, H)}{H+1} \approx \alpha$, there exist $c, a \in[0, H]$ such that

$$
\frac{c}{H+1} \approx 0, \frac{{ }^{*} A(a+1, H)}{H-a} \approx 1, \text { and }{ }^{*} A \cap[c, a]=\emptyset .
$$

Then the conclusion of Part II of Theorem 1.3 is true.

Proof. Let $\left\langle b_{n}: n \in \mathbb{N}\right\rangle$ be an increasing sequence in $\mathbb{N}$ such that

$$
\lim _{n \rightarrow \infty} \frac{A\left(0, b_{n}\right)}{b_{n}+1}=\alpha
$$

Then one has

$$
\frac{{ }^{*} A\left(0, b_{N}\right)}{b_{N}+1} \approx \alpha
$$

for each hyperfinite integer $N$. By the assumption of the lemma, there exist $c, a \in$ $\left[0, b_{N}\right]$ such that

$$
\frac{c}{b_{N}+1} \approx 0, \frac{{ }^{*} A\left(a+1, b_{N}\right)}{b_{N}-a} \approx 1, \text { and }{ }^{*} A \cap[c, a]=\emptyset .
$$

This means that for each $k \in \mathbb{N}$ and each hyperfinite $K$, the sentence $\varphi\left({ }^{*} A,{ }^{*} \mathbb{N}, K, k,\left\langle b_{n}: n \in{ }^{*} \mathbb{N}\right\rangle\right)$ is true in ${ }^{*} V$, where

$$
\begin{aligned}
\varphi\left({ }^{*} A,\right. & \left.\mathbb{N}, K, k,\left\langle b_{n}: n \in{ }^{*} \mathbb{N}\right\rangle\right) \\
& =\left(\forall N \in{ }^{*} \mathbb{N}\right)\left(N>K \text { implies }\left(\exists a_{N, k}, c_{N, k} \in{ }^{*} \mathbb{N}\right)\right) \\
& \left(\frac{c_{N, k}}{b_{N}+1}<\frac{1}{k} \text { and } \frac{{ }^{*} A\left(a_{N, k}+1, b_{N}\right)}{b_{N}-a_{N, k}}>1-\frac{1}{k} \text { and }{ }^{*} A \cap\left[c_{N, k}, a_{N, k}\right]=\emptyset\right) .
\end{aligned}
$$

By the underspill principle and the transfer principle, there is an $l_{k} \in \mathbb{N}$ such that $\varphi\left(A, \mathbb{N}, l_{k}, k,\left\langle b_{n}: n \in \mathbb{N}\right\rangle\right)$ is true in the standard world. Without loss of generality, we choose $l_{k}$ as an increasing sequence for $k=1,2, \ldots$. For each $n \in\left[l_{k}+1, l_{k+1}\right]$, let $a_{n}=a_{n, k}$ and $c_{n}=c_{n, k}$. For each $n \leqslant l_{1}$, let $a_{n}=c_{n}-1=b_{n}$. It is easy now to verify that the sequences $\left\langle a_{n}: n \in \mathbb{N}\right\rangle$ and $\left\langle c_{n}: n \in \mathbb{N}\right\rangle$ are what we are looking for. $\square($ Lemma 2.8$)$

The next lemma is just an observation and will be used frequently in the proof of the theorem.

Lemma 2.9. Let $A$ be such that $\bar{d}(A)=\alpha$ and let $H$ be hyperfinite such that $\frac{{ }^{*} A(0, H)}{H+1} \approx \alpha$. Suppose $X={ }^{*} A \cap[0, H]$ and $Y=\{H-a: a \in X\}$. If $0 \prec b \prec H$, then

$$
\frac{Y(0, b)}{b+1} \gtrsim \alpha
$$


(ii)

(iii)

$$
\frac{Y(0, b)}{b+1} \approx \alpha \text { implies } \frac{Y(b+1, H)}{H-b} \approx \alpha,
$$

$$
\frac{Y(0, b)}{b+1} \gg \alpha \text { implies } \frac{Y(b+1, H)}{H-b} \ll \alpha .
$$

Proof. Part (i) is true because otherwise $\frac{{ }^{*} A(0, H-b-1)}{H-b} \gg \alpha$, which implies $\bar{d}(A)>\alpha$ by Lemma 2.4. The conclusions (ii) and (iii) are true because $\frac{Y(0, H)}{H+1} \approx \alpha$ and one of $\frac{Y(0, b)}{b+1}$ or $\frac{Y(b+1, H)}{H-b}$ is $\gg \alpha$, which implies that the other has to be $\ll \alpha$. $\square$ (Lemma 2.9)

\section{Proof of Theorem 1.3$]$ and Corollary 1.4}

Now we are ready to prove Theorem [1.3. The proof of Part I of Theorem [1.3] is easy and the proof of Part II is hard and tedious.

Proof of Theorem 1.3. Let $A$ be a set with $\bar{d}(A)=\alpha$. Without loss of generality, we assume $0 \in A$. Let $H$ be a hyperfinite integer with $\frac{{ }^{*} A(0, H)}{H+1} \approx \alpha$. Again without loss of generality, we assume $H \in{ }^{*} A$ because otherwise we can just replace $H$ by the largest element in ${ }^{*} A \cap[0, H]$ and the interval excluded is insignificant relative to $H$. Let $X={ }^{*} A \cap[0, H]$ and let $Y=\{H-a: a \in X\}$. Then $|2 Y+\{-1,0\}|=$ $|2 X+\{0,1\}|$ and $(2 Y+\{-1,0\})(a, b)=(2 X+\{0,1\})(2 H-b, 2 H-a)$ for any $a, b \in[0,2 H]$.

Proof of Part I of Theorem 1.3. Let $\alpha \geqslant \frac{1}{2}$ and $\bar{d}(2 A+\{0,1\})=\frac{1+\alpha}{2}$. By Lemma 2.7, it suffices to show that

$$
\frac{(2 Y+\{-1,0\})(H+1,2 H)}{H+1} \approx \alpha .
$$

For notational convenience, we replace $\{-1,0\}$ by $\{0,1\}$. By a remark right after Lemma 1.1, one has

$$
\frac{(2 Y+\{0,1\})(0,2 H)}{2 H+1} \gtrsim \frac{1+\alpha}{2}
$$

because

$$
\frac{Y(0, H)}{H+1} \approx \alpha \geqslant \frac{1}{2} .
$$

So

$$
\frac{(2 Y+\{0,1\})(0,2 H)}{2 H+1} \approx \frac{1+\alpha}{2}
$$

by Lemma 2.4. By Lemma 2.5

$$
\frac{(2 Y+\{0,1\})(0, H)}{H+1} \approx 1 .
$$

Hence

$$
\begin{aligned}
\frac{1+\alpha}{2} & \approx \frac{(2 Y+\{0,1\})(0, H)+(2 Y+\{0,1\})(H+1,2 H)}{2 H+1} \\
& \approx \frac{1}{2}+\frac{1}{2} \cdot \frac{(2 Y+\{0,1\})(H+1,2 H)}{H+1}
\end{aligned}
$$


and this implies

$$
\frac{(2 Y+\{0,1\})(H+1,2 H)}{H+1} \approx \alpha .
$$

$\square($ Part I)

Proof of Part II of Theorem 1.3. Let $0<\alpha<\frac{1}{2}$ and $\bar{d}(2 A+\{0,1\})=\frac{3}{2} \alpha$. By Lemma 2.8 it suffices to show that there are $\bar{a}, \bar{c} \in[0, H]$ such that

$$
\frac{Y(0, \bar{a})}{\bar{a}+1} \approx 1, \frac{H-\bar{c}}{H+1} \approx 0, \text { and } Y \cap[\bar{a}+1, \bar{c}]=\emptyset .
$$

Again without loss of generality, we replace $\{-1,0\}$ by $\{0,1\}$ for notational convenience. Note that

$$
\frac{(2 Y+\{0,1\})(0,2 H)}{2 H+1} \approx \frac{3}{2} \alpha
$$

by Lemma 1.1 and Lemma 2.4 We will prove Part II by eliminating all other possibilities for $Y$ except the one we precisely want. We will do so in the next five claims. The main argument in the proofs of the first four claims can be stated roughly as follows. Suppose $Y$ is not what we want. Then we find a proper interval $[0, a]$ with $0 \prec a \prec H$ such that if $a \leqslant H-a$, then $(2 Y+\{0,1\})(0,2 a)$ is four times more than $Y(0, a)$ and $(2 Y+\{0,1\})(2 a+1,2 H)$ is three times more than $Y(a+1, H)$. Hence $\frac{(2 Y+\{0,1\})(0,2 H)}{2 H+1} \gg \frac{3 Y(0, H)}{2 H+1} \approx \frac{3}{2} \alpha$. If $a>H-a$, then $(2 Y+\{0,1\})(0, H)$ is significantly two times more than $Y(0, H)$ and $(2 Y+\{0,1\})(H, 2 H)$ is more than or equal to $Y(0, H)$. Hence $\frac{(2 Y+\{0,1\})(0, H)}{2 H+1} \gg \frac{2 Y(0, H)}{2 H+1}$ and $\frac{(2 Y+\{0,1\})(H, 2 H)}{2 H+1} \gtrsim \frac{Y(0, H)}{2 H+1}$. So $\frac{(2 Y+\{0,1\})(0,2 H)}{2 H+1} \gg \frac{3 Y(0, H)}{2 H+1} \approx \frac{3}{2} \alpha$.

Claim 1.3.1. The following sentence $\varphi$ is false, where

$$
\varphi=(\forall a \succ 0)(\exists b \succ 0)\left(b \leqslant a \leqslant H \text { and } \frac{Y(0, b)}{b+1} \approx \alpha\right) .
$$

Proof of Claim 1.3.1. Suppose the sentence is true. Choose a $b \succ 0$ such that

$$
2 b<H, b+1 \in Y, \text { and } \frac{Y(0, b)}{b+1} \approx \alpha .
$$

Then for each $x$ with $0 \prec x \leqslant b$,

$$
\frac{Y(0, x)}{x+1} \gtrsim \alpha
$$

and for each $x$ with $b \prec x \leqslant 2 b+1$,

$$
\frac{Y(b+1, x)}{x-b} \gtrsim \alpha .
$$


By Lemma 2.5 and Lemma 1.1 one has

$$
\begin{aligned}
& \frac{(2 Y+\{0,1\})(0,2 H)}{2 H+1} \\
& =\frac{(2 Y+\{0,1\})(0, b)+(2 Y+\{0,1\})(b+1,2 b+1)+(2 Y+\{0,1\})(2 b+2,2 H)}{2 H+1} \\
& \geqslant \frac{(2(Y \cap[0, b])+\{0,1\})(0, b)}{2 H+1} \\
& +\frac{(Y \cap[0, b]+Y \cap[b+1,2 b+1]+\{0,1\})(b+1,2 b+1)}{2 H+1} \\
& +\frac{(2(Y \cap[b+1, H])+\{0,1\})(2 b+2,2 H)}{2 H+1} \\
& \gtrsim 2 \alpha \frac{b+1}{2 H+1}+2 \alpha \frac{b+1}{2 H+1}+3 \alpha \frac{H-b}{2 H+1} \\
& \gg \frac{3}{2} \alpha \text {, }
\end{aligned}
$$

a contradiction. $\square$ (Claim 1.3.1)

By Claim 1.3.1, we can now assume that there is an $a$ with $0 \prec a \leqslant H$ such that for each $b$ with $0 \prec b \leqslant a$, one has $\frac{Y(0, b)}{b+1} \gg \alpha$.

Claim 1.3.2. The following sentence $\varphi$ is false, where

$$
\varphi=\exists a\left(0 \prec a \prec H \text { and } \frac{Y(0, a)}{a+1} \approx \alpha\right) .
$$

Proof of Claim 1.3.2. First we can choose $a$ with $0 \prec a \prec H$ such that

$$
a+1 \in Y, \quad \frac{Y(0, a)}{a+1} \approx \alpha,
$$

and for each $x$ with $0 \prec x \prec a$,

$$
\frac{Y(0, x)}{x+1} \gg \alpha
$$

One can find such an $a$ in the intersection of a sequence of intervals $\bigcap_{i=1}^{\infty}\left[b_{i}, c_{i}\right]$ such that

$$
\frac{Y\left(0, c_{i}\right)}{c_{i}+1} \approx \alpha, \quad \frac{c_{i}-b_{i}}{H+1}<\frac{1}{i},
$$

and for each $x$ with $0 \prec x \prec b_{i}$,

$$
\frac{Y(0, x)}{x+1} \gg \alpha
$$

By Lemma [2.9] one has that for each $c$ with $a \prec c \leqslant H, \frac{Y(a+1, c)}{c-a} \gtrsim \alpha$. We now derive a contradiction by showing that

$$
\frac{Y(0,2 H)}{2 H+1} \gg \frac{3}{2} \alpha \text {. }
$$

Case 1: $2 a \preceq H$. Without loss of generality, we can assume that $2 a \leqslant H$ because otherwise $2 a-H$ is insignificant with respect to $H$. Then by the same 
proof as in the Claim 1.3.1 one has

$$
\begin{aligned}
& \frac{(2 Y+\{0,1\})(0,2 H)}{2 H+1} \\
& =\frac{(2 Y+\{0,1\})(0, a)+(2 Y+\{0,1\})(a+1,2 a+1)+(2 Y+\{0,1\})(2 a+2,2 H)}{2 H+1} \\
& \approx \frac{4 \alpha(a+1)+3 \alpha(H-a)}{2 H+1} \\
& \gg \frac{3}{2} \alpha \text {. }
\end{aligned}
$$

Case 2: $2 a \succ H$. Then by Lemma 2.5, one has

$$
\frac{(Y \cap[0, H-a-1]+Y \cap[a+1, H]+\{0,1\})(a+1, H)}{H-a} \gg 2 \alpha
$$

because of the choice of $a$ and $H-a-1 \prec a$. Hence

$$
\begin{aligned}
& \frac{(2 Y+\{0,1\})(0,2 H)}{2 H+1} \\
& =\frac{(2 Y+\{0,1\})(0, a)+(2 Y+\{0,1\})(a+1, H)+(2 Y+\{0,1\})(H+1,2 H)}{2 H+1} \\
& \gg 2 \alpha \frac{a+1}{2 H+1}+2 \alpha \frac{H-a}{2 H+1}+\frac{Y(0, H)}{2 H+1} \\
& \approx \alpha+\frac{\alpha}{2}=\frac{3}{2} \alpha \text {. }
\end{aligned}
$$

$\square($ Claim 1.3.2)

By Claim 1.3.1 and Claim 1.3.2, we can assume that for each $a$ with $0 \prec a \prec H$, $\frac{Y(0, a)}{a+1} \gg \alpha$.

Claim 1.3.3. The following sentence $\varphi$ is false, where

$$
\varphi=(\exists a, b)\left(0 \prec a \prec b \prec H \text { and } \frac{Y(0, a)}{a+1} \ll \frac{1}{2} \text { and } \frac{Y(0, a)}{a+1} \ll \frac{Y(0, b)}{b+1}\right) .
$$

Proof of Claim 1.3.3. Suppose there exist $a$ and $b$, which witness that $\varphi$ is true. Let $-1=x_{0}<x_{1}<\cdots<x_{k}=b$ and $r_{0}, r_{1}, \ldots, r_{k-1}$ be two sequences constructed the same as in Lemma 2.3 with $[a, b]$ being replaced by $[0, b]$ and $A$ being replaced by $Y \cap[0, b]$.

Case 1: There is an $m \in[0, k]$ such that $x_{m} \succ 0$ and for each $i \in[0, m-1]$, $x_{i+1}-x_{i} \sim 0$.

Choose a $j \in[0, m-1]$ such that

$$
0 \prec x_{j} \prec a, \frac{Y(0, a)}{a-x_{j}} \ll \frac{1}{2}, \text { and } 2 x_{j}<x_{m} .
$$

Then for each $i<j$, one has $r_{i}<\frac{1}{2}$ by the choice of $x_{j}$ and the minimality of $r_{i}$. Note that

$$
Y\left(0, x_{j}\right)=\sum_{i=0}^{j-1} Y\left(x_{i}+1, x_{i+1}\right)=\sum_{i=0}^{j-1} r_{i}\left(x_{i+1}-x_{i}\right)
$$


Note also that for each $i \in[0, j-1], 2 x_{i+1}+1 \leqslant b$. Hence by Lemma [2.5 and Lemma 1.1, one has

$$
\begin{aligned}
&(2 Y+\{0,1\})(0,2 H) \\
&=(2 Y+\{0,1\})\left(0,2 x_{j}+1\right)+(2 Y+\{0,1\})\left(2 x_{j}+2,2 H\right) \\
& \geqslant\left(\sum_{i=0}^{j-1}(2 Y+\{0,1\})\left(2 x_{i}+2,2 x_{i+1}+1\right)\right)+(2 Y+\{0,1\})\left(2 x_{j}+2,2 H\right) \\
& \geqslant\left(\sum _ { i = 0 } ^ { j - 1 } \left(\left(Y \cap\left[x_{i}+1, x_{i+1}\right]+Y \cap\left[x_{i}+1, x_{i+1}\right]+\{0,1\}\right)\left(2 x_{i}+2, x_{i}+x_{i+1}+1\right)\right.\right. \\
&+\left(Y \cap\left[x_{i}+1, x_{i+1}\right]+Y \cap\left[x_{i+1}+1,2 x_{i+1}-x_{i}\right]\right. \\
&\left.\left.+\{0,1\})\left(x_{i}+x_{i+1}+2,2 x_{i+1}+1\right)\right)\right) \\
&+(2 Y+\{0,1\})\left(2 x_{j}+2,2 H\right) \\
& j-1 \\
& \geqslant\left(\sum_{i=0}^{j}\left(2 r_{i}\left(x_{i+1}-x_{i}\right)+2 r_{i}\left(x_{i+1}-x_{i}\right)\right)\right)+3 Y\left(x_{j}+1, H\right)-3 \\
&= 4 Y\left(0, x_{j}\right)+3 Y\left(x_{j}+1, H\right)-3 \\
&= 3 Y(0, H)+Y\left(0, x_{j}\right)-3 .
\end{aligned}
$$

Note that $\frac{Y\left(0, x_{j}\right)}{H+1} \gg 0$. Hence we have

$$
\frac{(2 Y+\{0,1\})(0,2 H)}{2 H+1} \geqslant \frac{3 Y(0, H)}{2 H+1}+\frac{Y\left(0, x_{j}\right)}{2 H+1}-\frac{3}{2 H+1} \gg \frac{3}{2} \alpha .
$$

Case 2: For every $m \in[0, k]$ with $x_{m} \succ 0$, there is an $i<m$ such that $x_{i+1}-x_{i} \succ$ 0 . Let

$$
t=\min \left\{\left(\frac{Y(0, b)}{b+1}-\frac{Y(0, a)}{a+1}\right),\left(\frac{1}{2}-\frac{Y(0, a)}{a+1}\right)\right\} .
$$

Then $t \gg 0$. Let $x \succ 0$ be such that

$$
\frac{Y(0, a)}{a-x} \ll \frac{Y(0, a)}{a+1}+\frac{t}{2}
$$

and let $x_{i_{0}}=\max \left\{x_{i}: x_{i}<x\right\}$. If $x_{i_{0}} \sim 0$, then $x_{i_{0}+1}-x_{i_{0}} \succ 0$. Let $j=i_{0}$. If $x_{i_{0}} \succ 0$, then there is a $j<i_{0}$ such that $x_{j+1}-x_{j} \succ 0$. By the choice of $x$, one has $r_{j} \ll \frac{1}{2}$. Since the sequence $r_{i}$ is nondecreasing by Lemma 2.3, then one has

$$
r_{j} \geqslant \frac{Y\left(0, x_{j+1}\right)}{x_{j+1}+1} \gg \alpha .
$$

Suppose $x_{j+1}-x_{j} \leqslant H-x_{j+1}$. Note that

$$
s=\min \left\{\frac{Y\left(x_{j+1}+1, x\right)}{x-x_{j+1}}: x \in\left[x_{j+1}+1, H\right]\right\} \gg 0
$$

and

$$
\begin{aligned}
& \frac{\left(2\left(Y \cap\left[x_{j}+1, x_{j+1}\right]\right)+\{0,1\}\right)\left(2 x_{j}+2, x_{j}+x_{j+1}+1\right)}{x_{j+1}-x_{j}} \\
& +\frac{\left(Y \cap\left[x_{j}+1, x_{j+1}\right]+Y \cap\left[x_{j+1}+1,2 x_{j+1}-x_{j}\right]+\{0,1\}\right)\left(x_{j}+x_{j+1}+2,2 x_{j+1}+1\right)}{x_{j+1}-x_{j}} \\
& \geqslant 2 r_{j}+r_{j}+s .
\end{aligned}
$$


Then by Lemma 1.1. Lemma 2.5, and the inequality above, one has

$$
\begin{aligned}
& \frac{(2 Y+\{0,1\})(0,2 H)}{2 H+1} \\
& \geqslant \frac{(2 Y+\{0,1\})\left(0,2 x_{j}+1\right)}{2 H+1} \\
&+\frac{(2 Y+\{0,1\})\left(2 x_{j}+2,2 x_{j+1}+1\right)}{2 H+1} \\
&+\frac{(2 Y+\{0,1\})\left(2 x_{j+1}+2,2 H\right)}{2 H+1} \\
& \geqslant \frac{3 Y\left(0, x_{j}\right)}{2 H+1}+\frac{\left(3 r_{j}+s\right)\left(x_{j+1}-x_{j}\right)}{2 H+1}+\frac{3 Y\left(x_{j+1}+1, H\right)}{2 H+1} \\
&= \frac{3 Y(0, H)}{2 H+1}+\frac{s\left(x_{j+1}-x_{j}\right)}{2 H+1} \gg \frac{3}{2} \alpha .
\end{aligned}
$$

Suppose $x_{j+1}-x_{j}>H-x_{j+1}$. Let $x_{j+1}=x_{0}^{\prime}<x_{1}^{\prime}<\cdots<x_{k^{\prime}}^{\prime}$ and $r_{0}^{\prime}, r_{1}^{\prime}, \ldots, x_{k^{\prime}-1}^{\prime}$ be two sequences constructed as in Lemma 2.3 with $[a, b]$ being replaced by $\left[x_{j+1}+1, H\right]$ and $A$ being replaced by $Y \cap\left[x_{j+1}+1, H\right]$. Note that for each $i \in\left[0, k^{\prime}-1\right]$ with $x_{i}^{\prime} \prec H$, one has $r_{i}^{\prime} \lesssim \alpha$. So $r_{i}^{\prime}+r_{j} \leqslant 1$. Since the first possible $i$ with $r_{i}^{\prime}+r_{j} \geqslant 1$ makes $x_{i}^{\prime} \sim H$, we can assume, without loss of generality, that $r_{i}^{\prime}+r_{j} \leqslant 1$ for every $i \in\left[0, k^{\prime}-1\right]$. By Lemma 2.2, one has

$$
\begin{aligned}
&\left(Y \cap\left[x_{j+1}+1, H\right]+Y\right.\left.\cap\left[x_{j}+1, H-x_{j+1}+x_{j}\right]+\{0,1\}\right)\left(x_{i}+x_{j+1}+2, H+x_{j}+1\right) \\
& \geqslant Y\left(x_{j+1}+1, H\right)+r_{j}\left(H-x_{j+1}\right) .
\end{aligned}
$$

Note that $r_{j} \gg \alpha \gtrsim \frac{Y\left(x_{j+1}+1, H\right)}{H-x_{j+1}}$. Hence

$$
\begin{aligned}
&\left.\frac{(2 Y}{}+\{0,1\}\right)(0,2 H) \\
& 2 H+1 \\
&= \frac{(2 Y+\{0,1\})\left(0,2 x_{j}+1\right)}{2 H+1} \\
&+\frac{(2 Y+\{0,1\})\left(2 x_{j}+2, x_{j+1}+x_{j}+1\right)}{2 H+1} \\
&+\frac{(2 Y+\{0,1\})\left(x_{j+1}+x_{j}+2, H+x_{j}+1\right)}{2 H+1} \\
&+\frac{(2 Y+\{0,1\})\left(H+x_{j}+2,2 H\right)}{2 H+1} \\
& \geqslant \frac{3 Y\left(0, x_{j}\right)-3}{2 H+1}+\frac{2 r_{j}\left(x_{j+1}-x_{j}\right)}{2 H+1} \\
&+\frac{Y\left(x_{j+1}+1, H\right)+r_{j}\left(H-x_{j+1}\right)}{2 H+1}+\frac{Y\left(x_{j}+2, H\right)}{2 H+1} \\
& \approx \frac{3 Y(0, H)}{2 H+1}+\frac{r_{j}\left(H-x_{j+1}\right)-Y\left(x_{j+1}+1, H\right)}{2 H+1} \\
& \gg \frac{3}{2} \alpha .
\end{aligned}
$$

\section{$\square($ Claim 1.3.3)}

Following the three claims above, we can now assume that for any $x, y$ with $0 \prec x \prec y \leqslant H$, either $\frac{Y(0, x)}{x+1} \gtrsim \frac{1}{2}$ or $\frac{Y(0, x)}{x+1} \gtrsim \frac{Y(0, y)}{y+1}$. From now on, we fix a 
$z \in[0, H]$ such that $\frac{Y(0, z)}{z+1} \approx \frac{1}{2}$ and for each $y$ with $z \prec y \leqslant H, \frac{Y(0, y)}{y+1} \ll \frac{1}{2}$. Note that $z \prec H$ because $\frac{Y(0, H)}{H+1} \approx \alpha \ll \frac{1}{2}$.

Claim 1.3.4. The following sentence $\varphi$ is false, where

$$
\varphi=\exists a(a+1 \in Y \text { and } z \prec a \prec H) .
$$

Proof of Claim 1.3.4. Suppose $\varphi$ is true and $a$ is a witness of the truth of $\varphi$. We divide the proof into three cases.

Case 1: $a \geqslant \frac{H}{2}$. Let $s=\frac{Y(0, a)}{a+1}$. Then $\frac{1}{2} \gg s \gg \alpha$. So

$$
\frac{Y(a+1, H)}{H-a} \ll \alpha .
$$

By Claim 1.3.3, for each $b$ with $0 \prec b \prec a$, one has

$$
\frac{Y(0, b)}{b+1} \gtrsim s
$$

Hence by Lemma 2.5, one has

$$
\frac{(2 Y+\{0,1\})(0, a)}{a+1} \gtrsim 2 s .
$$

Let $a=x_{0}<x_{1}<\cdots<x_{k}=H$ and $r_{0}, r_{1}, \ldots, r_{k-1}$ be two sequences constructed as in Lemma 2.3 with $[a, b]$ being replaced by $[a+1, H]$ and $A$ being replaced by $Y \cap[a+1, H]$. Note that $r_{i} \ll \alpha$ for each $x_{i} \prec H$ by the minimality of $r_{i}$. So $r_{i}+s<1$. Without loss of generality, we can assume that $r_{i}+s<1$ for every $i \in[0, k-1]$. By Lemma 2.2, one has

$$
\begin{gathered}
\frac{(Y \cap[a+1, H]+Y \cap[0, H-a-1]+\{0,1\})(a+1, H)}{H-a} \\
\gtrsim \frac{Y(a+1, H)+s(H-a)}{H-a} .
\end{gathered}
$$

Hence

$$
\begin{aligned}
& \frac{(2 Y+\{0,1\})(0,2 H)}{2 H+1} \\
& =\frac{(2 Y+\{0,1\})(0, a)+(2 Y+\{0,1\})(a+1, H)+(2 Y+\{0,1\})(H+1,2 H)}{2 H+1} \\
& \gtrsim \frac{2 Y(0, a)}{2 H+1}+\frac{(Y(a+1, H)+s(H-a))}{2 H+1}+\frac{Y(0, H)}{2 H+1} \\
& \approx \frac{3 Y(0, H)}{2 H+1}+\left(s-\frac{Y(a+1, H)}{H-a}\right) \frac{H-a}{2 H+1} \gg \frac{3}{2} \alpha .
\end{aligned}
$$

Case 2: $\quad a<\frac{H}{2}$ and $\frac{Y(a+1, H)}{H-a} \gg 0 . \quad$ Choose a $b \geqslant a$ such that $b+1 \in Y$ and for each $c$ with $b \prec c \leqslant H$, one has $\frac{Y(b+1, c)}{c-b} \gg 0$. If $b \geqslant \frac{H}{2}$, then the proof can be conducted exactly the same as the proof of Case 1 with $a$ being replaced by $b$. So we can assume that $b<\frac{H}{2}$. By Lemma 2.5, one has

$$
\frac{(Y \cap[b+1,2 b+1]+Y \cap[0, b]+\{0,1\})(b+1,2 b+1)}{b+1} \gg \frac{Y(0, b)}{b+1} .
$$


Hence

$$
\begin{aligned}
\frac{(2 Y+\{0,1\})(0,2 H)}{2 H+1} \\
\quad=\frac{(2 Y+\{0,1\})(0, b)+(2 Y+\{0,1\})(b+1,2 b+1)+(2 Y+\{0,1\})(2 b+2,2 H)}{2 H+1} \\
\quad \gg \frac{2 Y(0, b)}{2 H+1}+\frac{Y(0, b)}{2 H+1}+\frac{3 Y(b+1, H)}{2 H+1} \approx \frac{3}{2} \alpha .
\end{aligned}
$$

Case 3: $\quad a<\frac{H}{2}$ and $\frac{Y(a+1, H)}{H-a} \approx 0 . \quad$ Now

$$
\begin{aligned}
& \frac{(2 Y+\{0,1\})(0,2 H)}{2 H+1} \\
& =\frac{(2 Y+\{0,1\})(0, a)+(2 Y+\{0,1\})(a+1,2 a+1)+(2 Y+\{0,1\})(2 a+2,2 H)}{2 H+1} \\
& \gtrsim \frac{2 Y(0, a)+Y(0, a)+Y(0, a)}{2 H+1} \\
& =\frac{4 Y(0, a)}{2 H+1} \approx \frac{4 Y(0, H)}{2 H+1} \approx 2 \alpha \gg \frac{3}{2} \alpha .
\end{aligned}
$$

$\square($ Claim 1.3.4)

By the four claims above, we can assume that there is no $y \in Y$ such that $z \prec y \prec H$. Without loss of generality, we can assume that there is no $y \in Y$ with $z \leqslant y \prec H$. This clearly implies $z \succ 0$ and $\frac{z}{H+1} \approx 2 \alpha$. The proof of the next claim uses a completely different idea from the ideas in the proofs of the above four claims.

Claim 1.3.5. Let $a=\max (Y \cap[0, z])$. Then $a \sim\left\lceil\frac{z}{2}\right\rceil$.

Proof of Claim 1.3.5. First, we want to show that $(2 Y)(z, H) \sim 0$. Suppose this is not true. Then we have

$$
(2 Y)(0,2 H) \sim z+(2 Y)(z, H)+|H+Y| \sim \frac{3}{2} z+(2 Y)(z, H) \succ \frac{3}{2} z .
$$

This contradicts the fact that

$$
\frac{(2 Y)(0,2 H)}{2 H+1} \approx \frac{3}{2} \alpha .
$$

Then choose two integers $k<m$ in $\mathbb{N}$ such that $\frac{z}{m}<\frac{z}{k}<\frac{H-z}{2}$ and $k$ and $m$ are relatively prime.

For each $i=0,1, \ldots, 2 k$ let $x_{i}=\left\lceil i \frac{z}{2 k}\right\rceil$. Note that $x_{0}=0, x_{k}=\left\lceil\frac{z}{2}\right\rceil$, and $x_{2 k}=z$. Note also that for each $i \in[0,2 k-1], x_{i+1}-x_{i} \in\left\lceil\frac{z}{2 k}\right\rceil \pm\{0,1\}$. Let $c$ be such that $z+\left\lceil\frac{z}{k}\right\rceil+1<c \prec H$. Since

$$
\frac{(2 Y)(z, H)}{2 H+1} \approx 0
$$

and $2\left[x_{k}, x_{k+1}-1\right] \subseteq[z-2, c]$, then $Y\left(x_{k}, x_{k+1}-1\right) \sim 0$. For $i=1,2, \ldots, k-1$, since

$$
Y \cap\left[x_{k-i}, x_{k-i+1}-1\right]+Y \cap\left[x_{k+i}, x_{k+i+1}-1\right] \subseteq[z-2, c],
$$

then either both

$$
Y\left(x_{k-i}, x_{k-i+1}-1\right) \sim 0 \text { and } Y\left(x_{k+i}, x_{k+i+1}-1\right) \sim 0
$$


or one of the intervals $\left[x_{k-i}, x_{k-i+1}-1\right]$ and $\left[x_{k+i}, x_{k+i+1}-1\right]$ is disjoint from $Y$. Hence at least half of the intervals $\left[x_{i}, x_{i+1}-1\right]$ for $i=0,1, \ldots, 2 k-1$ satisfy

$$
\frac{Y\left(x_{i}, x_{i+1}-1\right)}{H+1} \approx 0 .
$$

Since $\frac{Y(0, z)}{z+1} \approx \frac{1}{2}$, then there are exactly half of $i \in[0,2 k-1]$ satisfying $\frac{Y\left(x_{i}, x_{i+1}-1\right)}{x_{i+1}-x_{i}}$ $\approx 0$ and the other half of $i \in[0,2 k-1]$ satisfying $\frac{Y\left(x_{i}, x_{i+1}-1\right)}{x_{i+1}-x_{i}} \approx 1$. So for each $i \in[0,2 k-1]$, either $\frac{Y\left(x_{i}, x_{i+1}-1\right)}{x_{i+1}-x_{i}} \approx 0$ or $\frac{Y\left(x_{i}, x_{i+1}-1\right)}{x_{i+1}-x_{i}} \approx 1$. Note that $\frac{Y\left(x_{0}, x_{1}-1\right)}{x_{1}-x_{0}} \approx 1$ because $\frac{Y\left(x_{k}, x_{k+1}-1\right)}{x_{k+1}-k_{i}} \approx 0$.

Now working exactly the same as the arguments above with $k$ being replaced by $m$, we have $2 m$ intervals $\left[y_{i}, y_{i+1}-1\right]$ for $i=0,1, \ldots, 2 m-1$ such that $y_{0}=0$, $y_{m}=\left\lceil\frac{z}{2}\right\rceil, z=y_{2 m}$, and for each $i \in[0,2 m-1], y_{i+1}-y_{i} \in\left\lceil\frac{z}{2 m}\right\rceil \pm\{0,1\}$. We have also that for each $i \in[0,2 m-1]$, either $\frac{Y\left(y_{i}, y_{i+1}-1\right)}{y_{i+1}-y_{i}} \approx 0$ or $\frac{Y\left(y_{i}, y_{i+1}-1\right)}{y_{i+1}-y_{i}} \approx 1$. Furthermore, there are exactly half of the intervals $\left[y_{i}, y_{i+1}-1\right]$ for $i=0,1, \ldots, 2 m-$ 1 satisfying each of the two conditions $\frac{Y\left(y_{i}, y_{i+1}-1\right)}{y_{i+1}-y_{i}} \approx 0$ and $\frac{Y\left(y_{i}, y_{i+1}-1\right)}{y_{i+1}-y_{i}} \approx 1$.

Since $k, m$ are relatively prime, one can show by the following argument that there are no $i \in[1, k-1]$ and $j \in[1, m-1]$ such that $x_{i} \sim y_{j}$.

Suppose

$$
\frac{\left\lceil i \frac{z}{2 k}\right\rceil-\left\lceil j \frac{z}{2 m}\right\rceil}{H} \approx 0
$$

for some $i \in[1, k-1]$ and $j \in[1, m-1]$. Then

$$
\frac{i \frac{z}{2 k}-j \frac{z}{2 m}}{H}=\frac{z}{H} \cdot \frac{i m-j k}{2 k m} \approx 0 .
$$

Since $\frac{z}{H} \gg 0$, then

$$
\frac{i m-j k}{2 k m} \approx 0
$$

This can be true only when $i m=j k$ because $2 m k$ is a standard integer. But $m i=j k$ contradicts that $k, m$ are relatively prime and $0<i<k$.

It is now clear that for all $i \in[0, k-1], \frac{Y\left(x_{i}, x_{i+1}-1\right)}{x_{i+1}-x_{i}} \approx 1$ because otherwise one can find a $j \in[1, m-2]$ with $\left[y_{j}, y_{j+1}-1\right]$ containing a significant portion of each of the two intervals $\left[x_{i}, x_{i+1}-1\right]$ and $\left[x_{i+1}, x_{i+2}-1\right]$ for some $i \in[0, k-2]$ with $\frac{Y\left(x_{i}, x_{i+1}-1\right)}{x_{i+1}-x_{i}} \approx 0$ and $\frac{Y\left(x_{i+1}, x_{i+2}-1\right)}{x_{i+2}-x_{i+1}} \approx 1$ or $\frac{Y\left(x_{i}, x_{i+1}-1\right)}{x_{i+1}-x_{i}} \approx 1$ and $\frac{Y\left(x_{i+1}, x_{i+2}-1\right)}{x_{i+2}-x_{i+1}} \approx 0$. But this is impossible because either $\frac{Y\left(y_{j}, y_{j+1}-1\right)}{y_{j+1}-y_{j}} \approx 0$ or $\frac{Y\left(y_{j}, y_{j+1}-1\right)}{y_{j+1}-y_{j}} \approx 1$ will lead to a contradiction.

Hence we have $Y\left(0,\left\lceil\frac{z}{2}\right\rceil\right) \sim\left\lceil\frac{z}{2}\right\rceil$. Since $a=\max (Y \cap[0, z])$, then $a \succ\left\lceil\frac{z}{2}\right\rceil$ or $a \sim\left\lceil\frac{z}{2}\right\rceil$. Suppose $a \succ\left\lceil\frac{z}{2}\right\rceil$. Then one has

$$
\begin{gathered}
(2 Y)(0,2 H) \succeq z+\mid\left(a+\left(Y \cap\left[0,\left\lceil\frac{z}{2}\right\rceil\right]\right) \cap[z, H]|+| H+Y \mid\right. \\
\sim 3 Y(0, H)+\mid\left(a+\left(Y \cap\left[0,\left\lceil\frac{z}{2}\right\rceil\right]\right) \cap[z, H] \mid \succ 3 Y(0, H),\right.
\end{gathered}
$$

which implies a contradiction that $\frac{(2 Y)(0,2 H)}{2 H+1} \gg \frac{3}{2} \alpha$. Hence we have $a \sim\left\lceil\frac{z}{2}\right\rceil$. $\square($ Claim 1.3.5) 
Now let $\bar{a}=a$ and let $\bar{c}=\min \{x \in Y: x>z\}-1$. Then $\bar{a}$ and $\bar{c}$ are the elements required at the beginning of the proof. $\square$ (Theorem [1.3)

Proof of Corollary 1.4 Note that $\underline{d}(A) \geqslant \beta$ if and only if for each hyperfinite integer $H$,

$$
\frac{{ }^{*} A(0, H)}{H+1} \gtrsim \beta .
$$

So we need only to show that there is a hyperfinite integer $K$ such that $\frac{{ }^{*} A(0, K)}{K+1} \approx 0$. If $\alpha<\frac{1}{2}$, then the conclusion is clear because by the proof of Part II of Theorem 1.3, one has

$$
0 \approx \frac{Y(\bar{c}, H)}{H-\bar{a}}=\frac{Y(\bar{a}+1, H)}{H-\bar{a}}=\frac{{ }^{*} A(0, H-\bar{a}-1)}{H-\bar{a}} .
$$

Suppose $\alpha \geqslant \frac{1}{2}$ and suppose $\underline{d}(A)=\beta>0$. Without loss of generality, we assume that $0 \in A$. Let $H$ be a hyperfinite integer such that $\frac{{ }^{*} A(0, H)}{H+1} \approx \alpha$ and let $\gamma$ be the greatest standard real number such that $\frac{{ }^{*} A(0, K)}{K+1} \gtrsim \gamma$ for each $K$ with $0 \prec K \leqslant H$. Note that $1>\alpha \geqslant \gamma \geqslant \beta$ and note that $2\left({ }^{*} A\right)+\{0.1\} \supseteq{ }^{*} A$. By Part I of Theorem 1.3, one has

$$
\frac{\left(2\left({ }^{*} A\right)+\{0,1\}\right)(0, K)}{K+1} \approx \frac{{ }^{*} A(0, K)}{K+1}
$$

for each $K$ with $0 \prec K \leqslant H$. However, by Lemma 2.5 one has

$$
\frac{\left(2\left({ }^{*} A\right)+\{0,1\}\right)(0, K)}{K+1} \gtrsim \min \{1,2 \gamma\}
$$

for each $K$ with $0 \prec K \leqslant H$. This contradicts the definition of $\gamma . \quad \square$ (Corollary 1.4)

\section{Remarks and Questions}

Remarks: (1) In Part I of Theorem 1.3 one cannot have a similar conclusion as in Part II. For example, let

$$
A=\bigcup_{n=2}^{\infty}\left(\left[\frac{1}{4} \cdot 2^{2^{n}}, \frac{3}{8} \cdot 2^{2^{n}}\right] \cup\left[\frac{1}{2} \cdot 2^{2^{n}}, 2^{2^{n}}\right]\right) .
$$

Then $\bar{d}(A)=\frac{5}{8}$

$$
\bar{d}(2 A+\{0,1\})=\frac{1+\frac{5}{8}}{2}=\frac{13}{16},
$$

and $A \cap\left[0,2^{2^{n}}\right]$ is not essentially concentrated on an interval $\left[a_{n}, 2^{2^{n}}\right]$ of length close to $\frac{5}{8} n$.

(2) In part II of Theorem 1.3 the term $\{0,1\}$ is not needed in the case of $\alpha>\frac{1}{2}$. This is because $(2 Y)(0,2 H) \supseteq[a, 2 H]$ for any $a$ with $H \prec a \prec 2 H$.

(3) By Example 1.2, we can see that the conclusions in Theorem 1.3 are reasonably optimal. However, the converses are not true. For example, let $E$ be the set of all even numbers and let

$$
A=\bigcup_{n=2}^{\infty}\left(\left[\frac{3}{4} \cdot 2^{2^{2 n}}, 2^{2^{2 n}}\right] \cup\left(\left[\frac{3}{5} \cdot 2^{2^{2 n+1}}, 2^{2^{2 n+1}}\right] \cap E\right)\right) .
$$


Then $\bar{d}(A)=\frac{1}{4}$ and the conclusion of Part II of Theorem[1.3 is true for $A$. However,

$$
\bar{d}(2 A+\{0,1\}) \geqslant \bar{d}(2 A) \geqslant \frac{4}{10}>\frac{3}{2} \cdot \frac{1}{4}=\frac{3}{8}=\frac{3}{2} \bar{d}(A) .
$$

In Theorem 1.3 the term $\{0,1\}$ is added for avoiding the trivial example of $A$ being an arithmetic sequence. The reader may feel that adding the term $\{0,1\}$ is not quite natural as a traditional condition when $0<\bar{d}(A) \leqslant \frac{1}{2}$. The traditional condition for $A$ should probably be $0 \in A$ and $\operatorname{gcd}(A)=1$ as in Freiman's theorem (Lemma 1.1). However, the following example shows that the above condition is not enough.

Example 5.1. Let $n \geqslant 4$ and $B=\{k n: k \in \mathbb{N}\}$. Let $m<n$ be a number such that $m$ and $n$ are relatively prime. Let $A=B \cup(B+m)$. Then one has $\bar{d}(A)=\frac{2}{n}, \bar{d}(2 A)=\frac{3}{n}=\frac{3}{2} \bar{d}(A), 0 \in A$, and $\operatorname{gcd}(A)=1$. Clearly, $A$ does not have the structural property described in the conclusion of Part II of Theorem 1.3 and the conclusion of Part I of Theorem 1.3 for the case of $\bar{d}(A)=\frac{1}{2}$.

Hence we would like to end this paper by asking the following question.

Question 5.2. Can Theorem 1.3 still be true in the case of $\bar{d}(A) \leqslant \frac{1}{2}$ if one omits the term $\{0,1\}$, adds the conditions $0 \in A$ and $\operatorname{gcd}(A)=1$, and assumes that $A$ is not the union of two arithmetic (infinite) sequences with the same common difference?

I conjecture that the answer is YES.

\section{REFERENCES}

[1] Bilu, Y., Addition of sets of integers of positive density, Journal of Number Theory, 64 (1997), No. 2, 233-275. MR 98e:11013

[2] Halberstam, H. and Roth, K. F., Sequences, Oxford University Press, 1966. MR 35:1565

[3] Hegedüs, P., Piroska, G., and Ruzsa, I. Z., On the Schnirelmann density of sumsets, Publ. Math. Debrecen, 53/3-4 (1998), 333-345. MR 99k:11019

[4] Henson, C. W., Foundations of nonstandard analysis: A gentle introduction to nonstandard extensions, in Nonstandard Analysis: Theory and Applications, ed. by N. J. Cutland, C. W. Henson, and L. Arkeryd, Kluwer Academic Publishers, Dordrecht, 1997. MR 99i:03085

[5] Jin, Renling, Nonstandard methods for upper Banach density problems, Journal of Number Theory 91 (2001), No. 1, 20-38. MR 2002h:11011

[6] Jin, Renling, Standardizing nonstandard methods for upper Banach density problems, to appear, DIMACS series for the workshop on Unusual application of number theory, Jan. 2000.

[7] Lindstrøm, T., An invitation to nonstandard analysis, in Nonstandard Analysis and its Applications, ed. by N. Cutland, Cambridge University Press, 1988. CMP 21:05

[8] Nathanson, Melvyn B., Additive Number Theory-Inverse Problems and the Geometry of Sumsets, Springer, 1996.

Department of Mathematics, College of Charleston, Charleston, South Carolina 29424

E-mail address: jinr@cofc.edu 